\title{
HIGH RESOLUTION OBSERVATIONS OF GENERALIZED FAST DRIFT BURSTS
}

\author{
ØYSTEIN ELGARøY and PER H. ROSENKILDE \\ Institute of Theoretical Astrophysics, Oslo University, Norway
}

\begin{abstract}
Solar Phys.). During 1969 and 1970 groups of generalized fast drift bursts were observed on 21 days with a high resolution radio spectrograph at Oslo Solar Observatory. Totally 48 groups were detected in the frequency band $310-340 \mathrm{MHz}$. In the great majority of the cases the groups were accompanied by metre wave type III bursts at lower frequencies.
\end{abstract}

In Figure 1 the distribution of durations of single bursts as measured at a chosen frequency $(318 \mathrm{MHz})$ is shown. The average burst duration amounted to $0.26 \mathrm{~s}$ in a sample comprising 834 bursts. For normal type III bursts observed in the same frequency band the average duration is $1 \mathrm{~s}$ (Elgarøy and Lyngstad, 1972).

Using data on the location of emissive regions at $169 \mathrm{MHz}$ and at $408 \mathrm{MHz}$ and

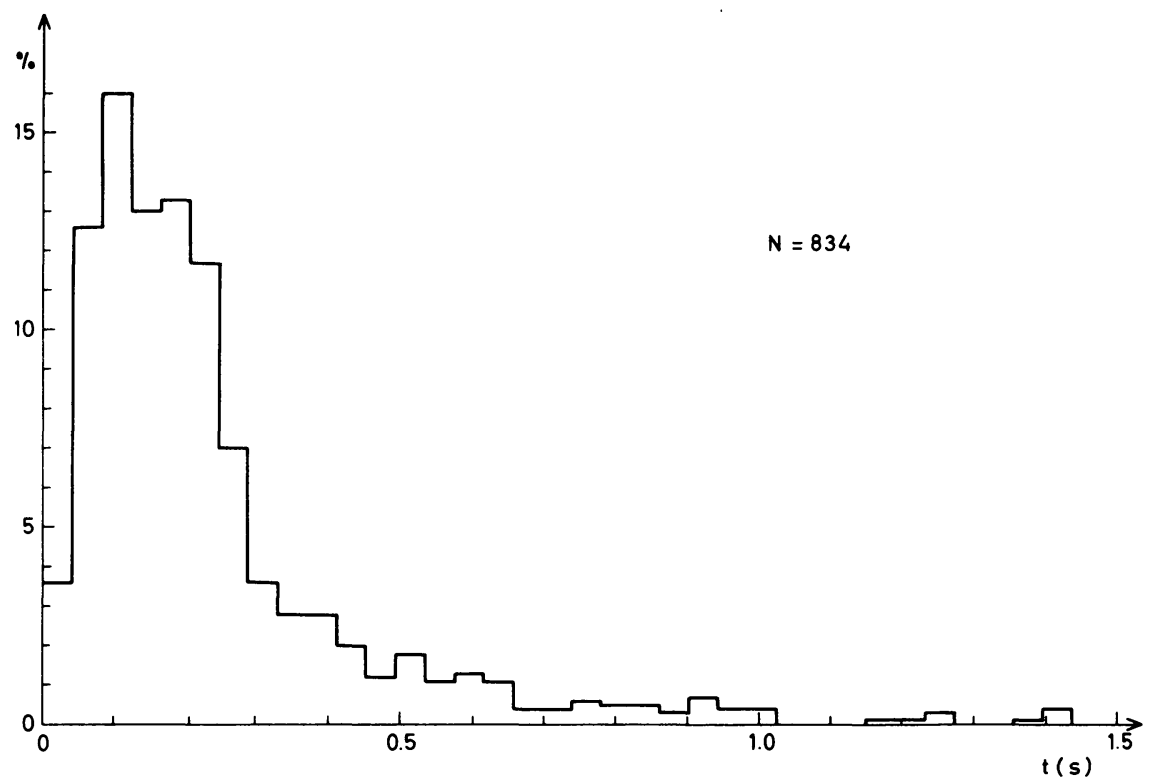

Fig. 1. Distribution of burst durations.

also data on the position of type III groups at $169 \mathrm{MHz}$ from Nançay, the position of the groups of generalized fast drift bursts on the solar disk has been estimated. Plotting the average burst duration against the distance of the source from the central meridian, it is found from Figure 2 that the duration increases with increasing distance from the centre. This may indicate the presence of scattering effects.

The frequency drift velocity was very high, and positive as well as negative drifts 
occurred. Frequently the starting time of a burst seemed to be the same at all frequencies. The distribution of measured values of inverse frequency drift velocities for 402 bursts is shown in Figure 3. In $50 \%$ of the cases the bursts had a negative drift, $17 \%$ of the bursts showed a positive drift, and for the last $33 \%$ of the bursts the fre-

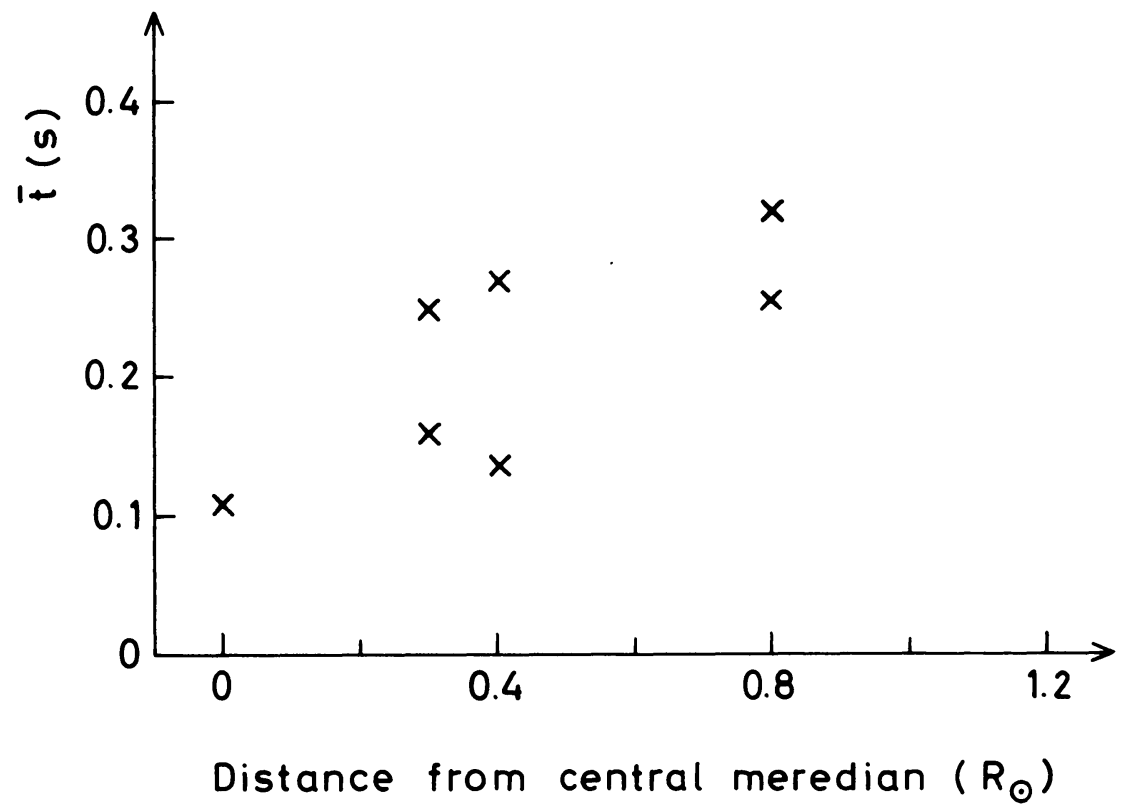

Fig. 2. Average burst duration at different distances from the central meredian.

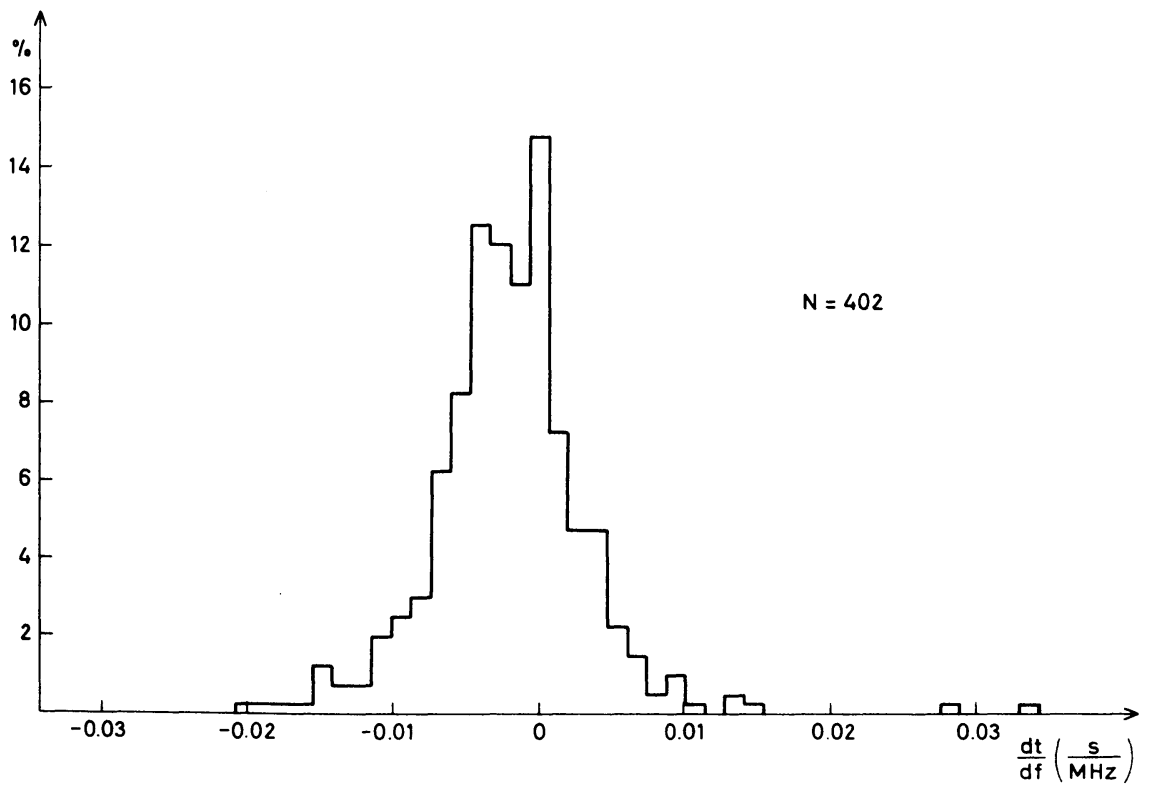

Fig. 3. Distribution of inverse frequency drift velocities $(\mathrm{d} t / \mathrm{d} f)$. 
quency drift was immeasurable $\left(|\mathrm{d} t / \mathrm{d} f|<0.002 \mathrm{~s} \mathrm{MHz}^{-1}\right)$. The distribution in Figure 3 is very different from the one found for metre wave type III bursts in the $300 \mathrm{MHz}$ range (Elgarøy and Lyngstad, 1972).

About $50 \%$ of the groups of generalized fast drift bursts occurred when Explorer 37 observed X-ray bursts from the Sun.

Generalized fast drift bursts strongly resemble some other types of short lasting bursts observed with the $310-340 \mathrm{MHz}$ radio spectrograph.

The fast drift bursts described here may be generated by plasma waves excited by particle streams directed towards the solar surface. The stream motion and differential group delay are then counteracting sources of frequency drift. When the first source dominates, positive frequency drift is observed whereas negative drift results when the situation is reversed. Zero frequency drift occurs when the two effects balance. The whole matter is a question of fractions of a second.

\section{Acknowledgements}

The authors are indebted to the solar group at Meudon for positional data on type III bursts at $169 \mathrm{MHz}$.

This research has been sponsored in part by the Air Force Cambridge Research Laboratories, United States Air Force under Grant AFOSR 72-2294.

\section{References}

Elgarøy, Ø. and Lyngstad, E.: 1972, Astron. Astrophys. 16, 1. 